\title{
A Comparative Study of Open Source Operating Systems for Virtualization with Executing Applications
}

\author{
Vikas Verma \\ Dept. of CSE \\ Lovely Professional University \\ Phagwara, Punjab
}

\author{
Sandeep Ranjan \\ Dept. of IT \\ KCL IMT \\ Jalandhar, Punjab
}

\author{
Yash Pal \\ Dept. of CSE \\ Lovely Professional University \\ Phagwara, Punjab
}

\begin{abstract}
Virtualization is becoming increasingly popular, both for desktop as well as server systems. It has great demand in Information Technology industry because of inherent benefits like physical resource sharing that result in better utilization and ease in managing the system. Several studies have been done to find out the impact that virtualization has on system performance. The experimentation on Virtualization is in use by educational institutions for research and teaching and this is being done at large level. This paper stresses on the comparison of execution time of two classical problem " $\mathrm{N}$ Queens problem $(\mathrm{N}=13)$ " and "Towers of Hanoi $(\mathrm{N}=20$ disks)" on various Linux distributions that are installed on VMWare Workstation 10.0. In this context, we keep the various parameters like disk size and sector size and memory size same for all the virtual guests.
\end{abstract}

\section{Keywords}

VMware Workstation, Virtual Machines, Operating System, N Queen Problem, Towers of Hanoi

\section{INTRODUCTION}

Virtualization is a way of presenting and using resources by function without regard to their physical layout or location. Every virtual machine (VM) is given the illusion that it is an obsessive physical machine that is totally protected and independent from alternative virtual machines. Virtual machines also are convenient abstractions of server workloads, since they cleanly encapsulate the complete state of a running system, as well as each user-level applications and kernel mode software system services [1]. In several computing environments, it is going to be the case that individual servers do not seem to be being used up to the mark that creates them to be thought-about or shapely as virtual machines on one physical server with very little or no performance penalty. Similarly, several tiny servers are combined onto fewer larger machines to simplify management and reduce costs. Ideally, system administrators ought to be able to flexibly over commit memory, processor, and alternative resources so as to reap the advantages of applied mathematics multiplexing, whereas still providing resource guarantees to VMs of varied importance [1].

Virtualization, quite an old concept was developed 50 years back in 1960's. This technology was initiated by IBM with the event of the IBM 360/67 and was designed to take full advantage of the process capabilities of costly mainframe systems which were divided into separate virtual machines. This configuration allowed multiple jobs to be executed at the same time as a method of investment for the expense of the hardware. In 1974, Popek and Goldberg outlined three characteristics of how virtual machines ought to behave.
These characteristics were associates in equivalence property (code executed on a virtual machine should execute in a standardized identical manner to code executing on hardware), a resource management property (the virtual machine ought to manage and protect all hardware resources), an efficiency property (safe instructions ought to be executed without intervention of the virtual machine) [2].

It is important to remark that the similarity level between the virtual and real atmosphere conjointly depends on the virtualization technique [3].

Even in universities with dedicated labs for courses, students seldom get opportunity to run their processes on multiple physical machines at the same time and observe the interaction among these processes [4]. For such situations, students may transfer pre-built Linux-based appliances (without any restriction on licensing moreover as comparatively lower resource overhead than Windows-based appliances) exploitation that they will at the same time run many virtual machines and check their applications. Virtual machines play a big role in reducing the requirement for many physical host machines to run multiple processes.

Each virtual machine may be a duplicate of the underlying physical machine and user's square measure given the illusion of running directly on the physical machine. Virtual machines additionally offer edges like isolation, resource sharing, and also the ability to run multiple flavors and configurations of operation systems with totally different set of code technology and configuration [4].

Virtual machines have been used for many years to permit multiple copies of doubtless totally different operating systems to run at the same time on one hardware platform [5]. A virtual machine monitor (VMM) may be a computer code layer that virtualizes hardware resources, commerce a virtual hardware interface that reflects the underlying machine design, as an example, the powerful VM/370 virtual machine system [6] supported multiple synchronic virtual machines, each one of which is believed to be running natively on the IBM System/370 hardware design [7]. More modern analysis, exemplified by dance palace, has targeted on exploitation virtual machines $[8,9]$ to supply quantifiability and fault containment for goods operative systems running on largescale shared memory multiprocessors.

Because virtual machines work by time-sharing host physical hardware, they cannot specifically duplicate the temporal arrangement activity of physical machines. VMware virtual machines use many techniques to reduce and conceal variations in temporal arrangement performance; however the variations will still generally cause activity inaccuracies and 
different issues in computer code running in every virtual machine. This data guide describes activity hardware works in physical machines, however typical guest operating systems use this hardware to keep time and the way VMware product virtualize the hardware [10].

VMware digital computer may be a piece of hardware, computer code or code that lets users to line up multiple virtual machines (VMs) and these machines are often used together with a particular laptop machine on which VMWare digital computer is put in. Every Virtual machine is capable of executing its own OS like UNIX operating system, Red Hat, Windows or Mac variants [11]. As such, VMware digital computer permits one physical machine to run multiple operating systems at the same time. Workstation is developed and sold-out by VMware, Inc., a division of EMC Corporation [11].

VMware digital computer supports bridging existing host network adapters and share physical disk drives and USB devices with a virtual machine. Additionally, it simulates disk drives and mounts associated existing ISO image file into a virtual memory device drive in order that the virtual machine sees it as a true one. Likewise, virtual disc drives are created via .vmdk files.

VMware digital computer can save the state of a virtual machine at any instance of time. These saved states, referred to as a "snapshots", will later be remodeled, effectively returning the virtual machine to the saved state [12]. VMware digital computer includes the power to designate multiple virtual machines as a team which may then be batterypowered on, battery-powered off, suspended or resumed as one object, creating it significantly helpful for testing clientserver environments.

\section{ADVANTAGES - VIRTUALIZATION}

There are several advantages to virtualizing server operating systems.

a. Multiple virtual operating systems: Instead of buying separate physical server to run each network operating System [2] and its applications, one physical server will run multiple virtual operating systems, reducing hardware prices, energy consumption, knowledge center house space, and disaster recovery expenses.

b. Live Migration: Another advantage of server virtualization is that it can be beneficial in providing uninterrupted server access to users. Data centers need to go for scheduled "downtime" for servers to perform maintenance of hardware or package [2]. However, with the quality and nearly unlimited access required for users, it's typically troublesome to search out a time once users won't be inconvenienced in that period. This could be self-addressed by virtualization that supports live migration. This technology allows a virtual machine to be stirred to another machine with no impact to the users. The virtual machine stores its current state onto a shared device straightaway before the migration happens. The virtual machine is then reinstalled on another machine and accesses its storage with no noticeable interruption to users. Live migration may be used for load balancing; if the demand for a service or application increases, then network managers will quickly move this high-demand virtual machine to a different physical server with additional RAM or hardware resources.

\section{AREA OF CONSIDERATION}

\subsection{8-Queen problem}

The 8-Queen problem is simple to describe:

\begin{tabular}{|c|l|}
\hline Given & An 8 by 8 chess board. \\
\hline Task & $\begin{array}{l}\text { Place } 8 \text { queens on the board so that they } \\
\text { do not attack each other. }\end{array}$ \\
\hline
\end{tabular}

Similarly, the problem of 10 -queen is to place 10 queens in such a way that they do not lie in same row or column or diagonal. The following figures $1 \mathrm{a}$ and $1 \mathrm{~b}$ shows solutions to 8 -queen problem. These figures represent two different solutions.

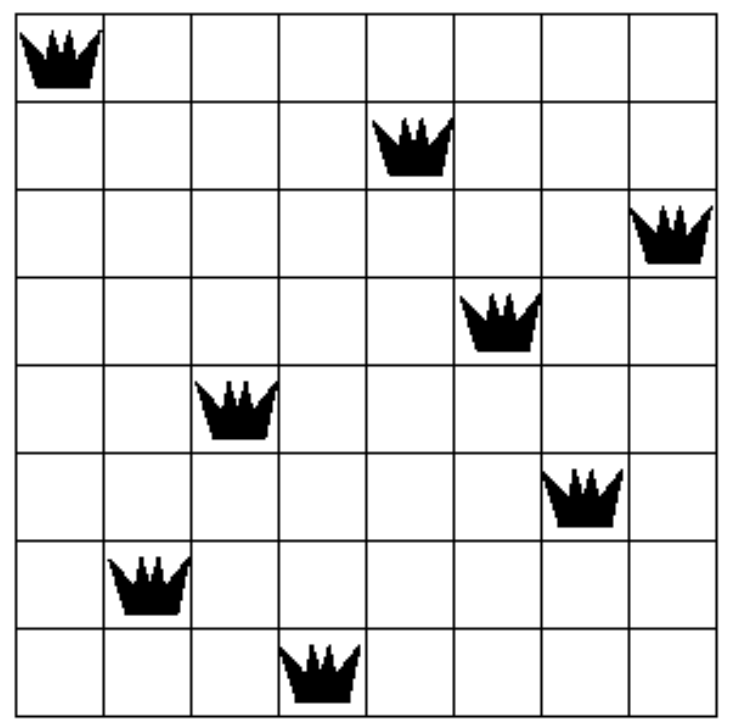

Fig 1a: Solution to 8-Queen problem

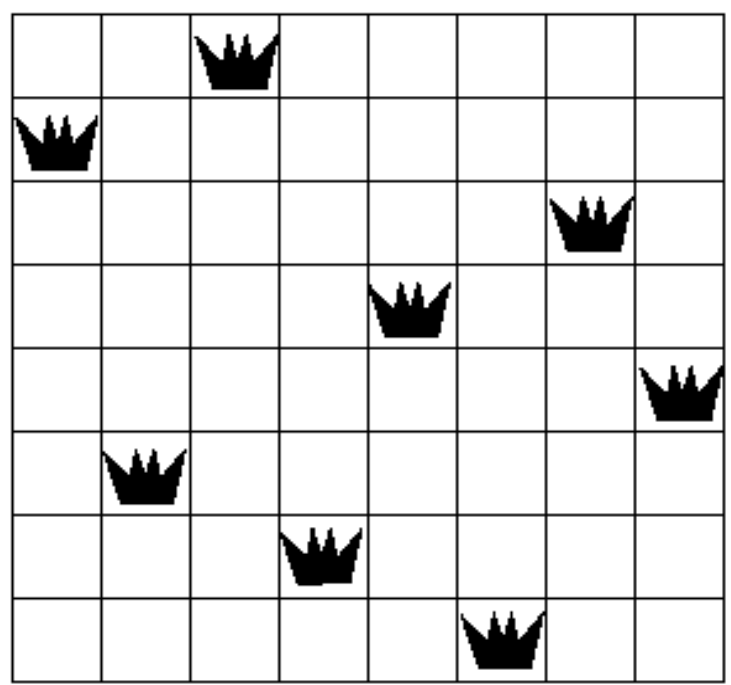

Fig 1b: Another solution to 8-Queen problem. 
Figure 2 shows some placement of queens for a solution to a 16-queens problem.

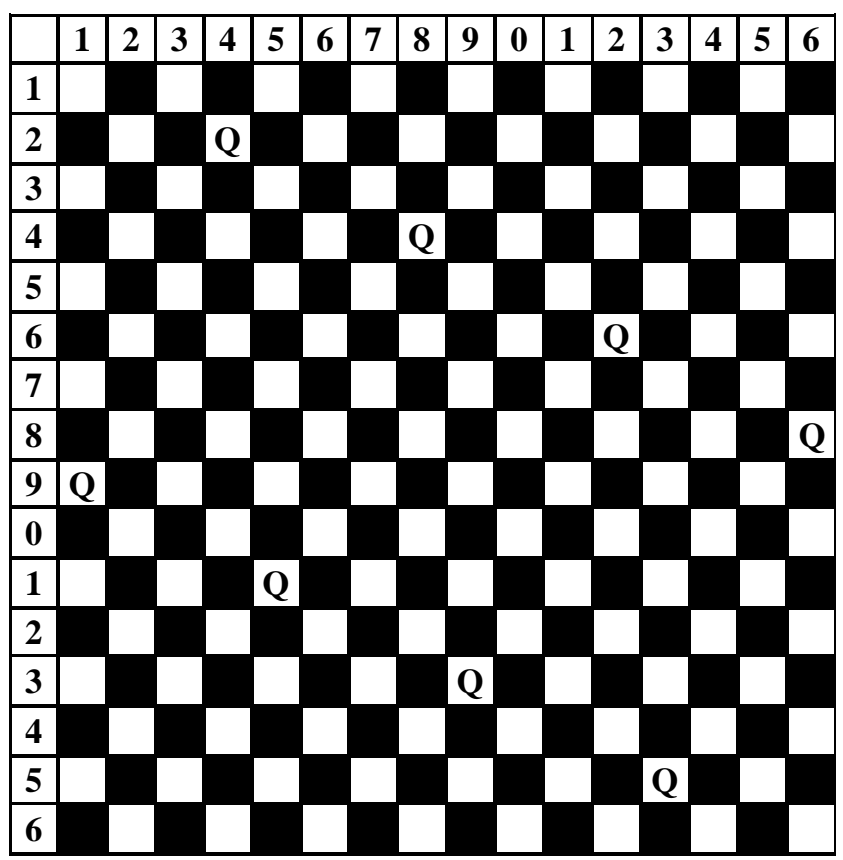

Fig 2: An approach to find solution to 16-Queens problem

\subsection{Tower of Hanoi}

Tower of Hanoi puzzle involves moving a pile of three different sized disks from one peg to another using an intermediate peg. Only one disk at a time can be moved, a disk can only be moved if it is the top disk on a pile, and a larger disk cannot be placed on a smaller one. The puzzle starts with the disks in a very neat stack in ascending order of size on one rod, the smallest at the top, thus making a conical shape.

The objective of the puzzle is to move the entire stack to another rod, obeying the following simple rules:

1. Only one disk can be moved at a time.

2. Each move consists of taking the upper disk from one of the stacks and placing it on top of another stack i.e. a disk can only be moved if it is the uppermost disk on a stack.

3. No disk may be placed on top of a smaller disk.

With three disks, the puzzle can be solved in seven moves. The minimum number of moves required to solve a Tower of Hanoi puzzle is $2 \mathrm{n}-1$, where $\mathrm{n}$ is the number of disks.

Figure 3 shows the initial and goal states of a three-disk problem.

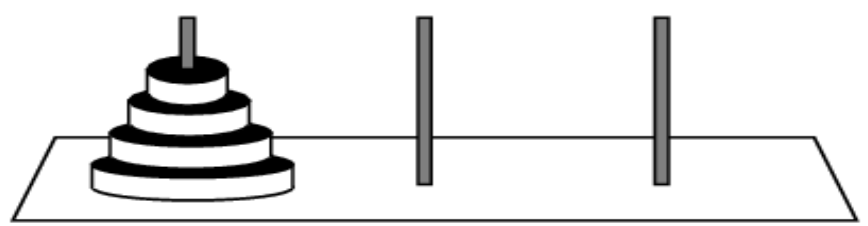

Fig 3: Tower of Hanoi problem

\section{EXPERIMEMT}

The experiments were conducted using a laptop configured with Intel Dual Core Processor 2.2 GHz, 3 GB RAM and 300 GB SATA hard disk. This physical machine has Windows7 32 bit as host OS and VMware 10 installed on it. We created virtual machines for Fedora 18, Fedora 19, Ubuntu 10, Ubuntu 13 and Red Hat 6. The main machine was rebooted before the beginning of each new set of tests.

The configuration of VMware guests was: - VMware workstations 10.0, 1 GB RAM 2 Processors and all other standard parameters for all guests.

The execution time of classical 8-Queen problem with 13 queens is observed as follows:-

The execution time of classical Towers of Hanoi problem with 20 disks is observed as follows:-

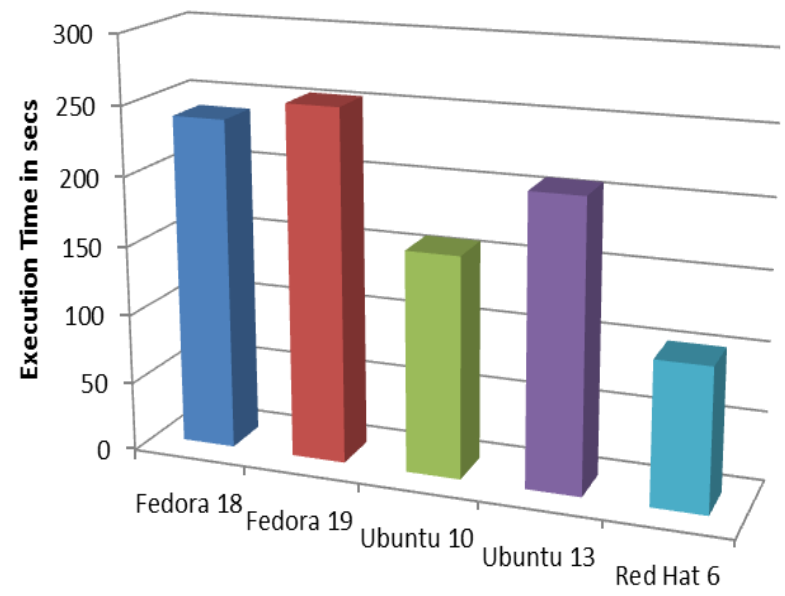

Fig 4: Execution time for 13-Queen problem

TABLE I

EXECUTION TIME FOR 13-QUEEN PROBLEM

\begin{tabular}{|c|c|}
\hline Operating System & Time (in secs) \\
\hline Fedora 18 & 235 \\
\hline Fedora 19 & 243 \\
\hline Ubuntu 10 & 231 \\
\hline Ubuntu 13 & 277 \\
\hline Red Hat 6 & 223 \\
\hline
\end{tabular}

TABLE II

EXECUTION TIME FOR TOWER OF HANOI PROBLEM

\begin{tabular}{|c|c|}
\hline Operating System & Time (in secs) \\
\hline Fedora 18 & 239 \\
\hline Fedora 19 & 254 \\
\hline Ubuntu 10 & 159 \\
\hline Ubuntu 13 & 208 \\
\hline Red Hat 6 & 103 \\
\hline
\end{tabular}




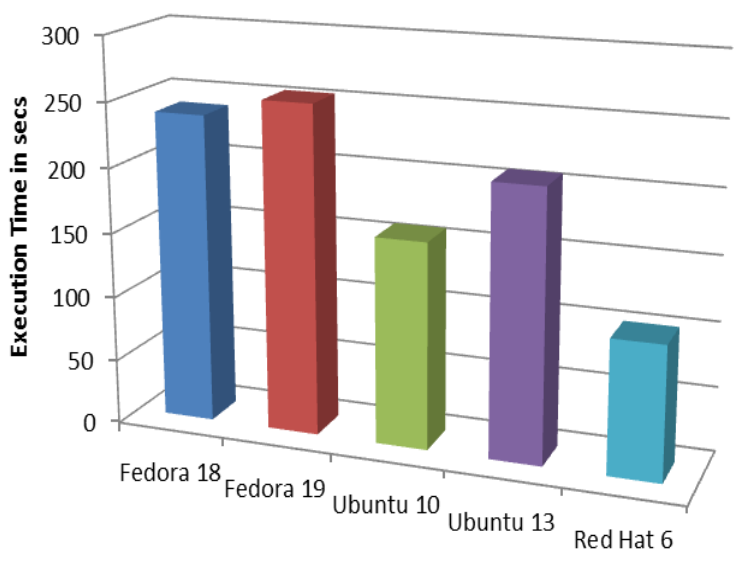

Fig 5: Execution time for Towers of Hanoi problem

\section{CONCLUSION AND FUTURE WORK}

The research work attempted to do a comparative analysis of three operating systems for execution time of two applications. In this paper, the performance of Fedora, Ubuntu and Red Hat as open source Linux versions operating systems for virtualization technology with execution of two major applications N-Queen problem and Towers of Hanoi have been evaluated. Our experimental results have showed that for both the problems, best solution is obtained through Red Hat 6. Our future studies include performance evaluations of execution time of more applications run on different guest operating system distributions of Linux with reference to Virtual Box to analyze the impact of virtualization software (VMware or Virtual Box) on the performance of guest operating system.

\section{REFERENCES}

[1] https://www.usenix.org/legacy/event/osdi02/tech/full_papers/waldsp-urger/waldspurger_html/node2.html.

[2] Mark Revels, Mark Ciampa, "Benchmarking Comparison of VMware Workstation and Sun VirtualBox OSE,"
[3] W. M. Fuertes, J. E. Lopez de Vergara, "A Quantitative Comparison of Virtual Network Envrionments based on Performance Measurements," Proceedings of the 14th HP Software University Association Workshop, Munich, Germany, July 2007.

[4] Ishtiaq Ali, Natarajan Meghanathan, "Virtual Machines and Networks - Installation, Performance, Study, Advantages and Virtualization Options," International Journal of Network Security \& Its Applications (IJNSA), Vol.3, No.1, January 2011, DOI 10.5121/ijnsa.2011.3101.

[5] Robert P. Goldberg. "Survey of Virtual Machine Research,” IEEE Computer, 7(6), June 1974.

[6] R. J. Creasy. "The Origin of the VM/370 Time-Sharing System," IBM Journal of Research and Development, 25(5), September 1981.

[7] Peter H. Gum. "System $/ 370$ Extended Architecture: Facilities for Virtual Machines," IBM Journal of Research and Development', 27(6), November 1983.

[8] Edouard Bugnion, Scott Devine, Kinshuk Govil, and Mendel Rosenblum. "Disco: Running Commodity Operating Systems on Scalable Multiprocessors," ACM Transactions on Computer Systems, 15(4), November 1997.

[9] Kinshuk Govil, Dan Teodosiu, Yongqiang Huang, and Mendel Rosenblum. "Cellular Disco: Resource Management Using Virtual Clusters on Shared-Memory Multiprocessors," Proc. Symposium on Operating System Principles, December 1999.

[10]

http://www.vmware.com/files/pdf/techpaper/Timekeepin g-In-Virtual-Machines.pdf

[11] http://en.wikipedia.org/wiki/VMware_Workstation.

[12] Warren, Steven (2008-11-20). "Snapshots in VMware Workstation". Blogs.techrepublic.com.com. Archived from the original on 2012-07-07. Retrieved 2012-09-13. 